\title{
DIALECTICS OF THE PAH ABUNDANCE TREND WITH METALLICITY
}

\author{
F. Galliano ${ }^{1}$
}

\begin{abstract}
This paper reviews the various processes that have been proposed to explain the observed trend of PAH strength with metallicity. It summarizes a study showing that, although PAH destruction by hard radiation is important in low-metallicity environments, it is not sufficient to explain their paucity. In these systems, the deficiency of their formation mechanism, related to stellar evolution, has to be invoked.
\end{abstract}

\section{Introduction: An intricate combination of processes}

From an extragalactic point of view, the strength of the aromatic features appears to be correlated with the metallicity of the environment. The general trend has been discussed by many authors. It was first demonstrated by Madden et al. (2006) using ISO spectra, and by Engelbracht et al. (2005) with Spitzer broadbands.

At the scale of a galaxy, the aromatic-feature-to-mid-IR-continuum intensity ratio appears to be a monotonic rising function of the metallicity of the gas, $Z_{\text {gas }}$. This relation contains a lot of scatter, and the metallicity is uncertain and sometimes difficult to define due to its inherent gradient within large galaxies. However, there is no reason to a priori consider that this trend defines two regimes above and below $12+\log (\mathrm{O} / \mathrm{H}) \simeq 8$. . The notion of such a threshold was introduced by Engelbracht et al. (2005), when studying the $\mathrm{IRAC}_{8} \mu \mathrm{m} / \mathrm{MIPS}_{24} \mu \mathrm{m}$ ratio. Galliano et al. (2008, Figure 1a of the present paper) showed that this was a bias due to the fact that, for low PAH-to-continuum ratios, the $\mathrm{IRAC}_{8} \mu \mathrm{m}$ band stops being a good tracer of the PAH strength, as it becomes dominated by the continuum intensity. With such a threshold, several galaxies, which are clearly PAH deficient, would fall in the regime where "normal" galaxies are.

Several scenarios have been proposed to explain the origin of the general trend.

1. PAHs are known to be massively destroyed in regions bathed with hard UV photons. The filling factor of molecular clouds in dwarf galaxies is lower

1 Service d'Astrophysique - Laboratoire AIM, CEA/Saclay, L'Orme des Merisiers, 91191 Gif-sur-Yvette, France; e-mail: frederic.galliano@cea.fr 

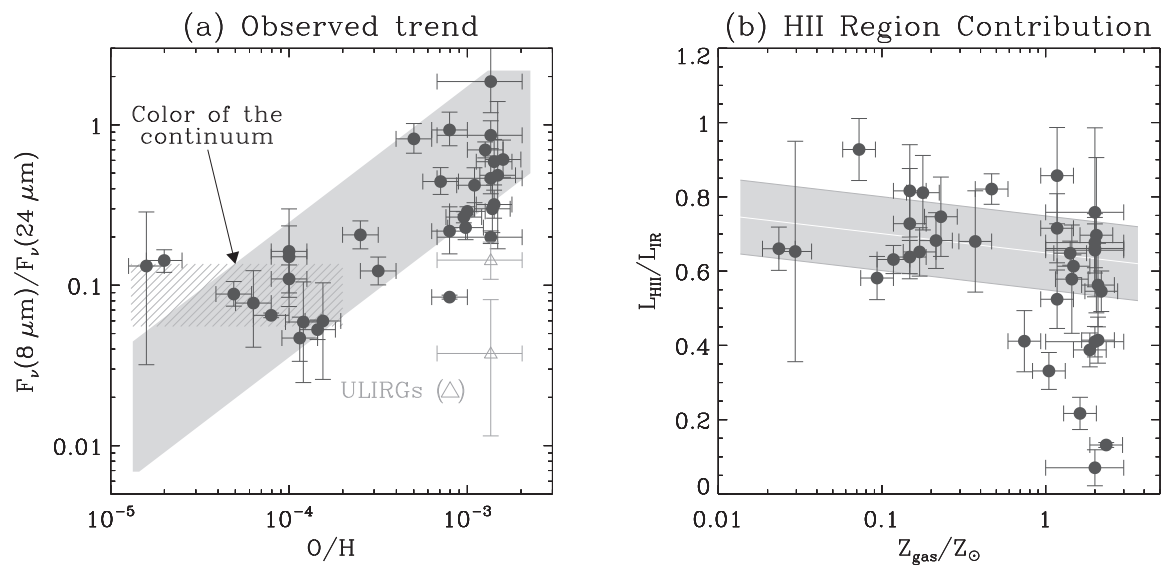

(c) Stellar Population Ages

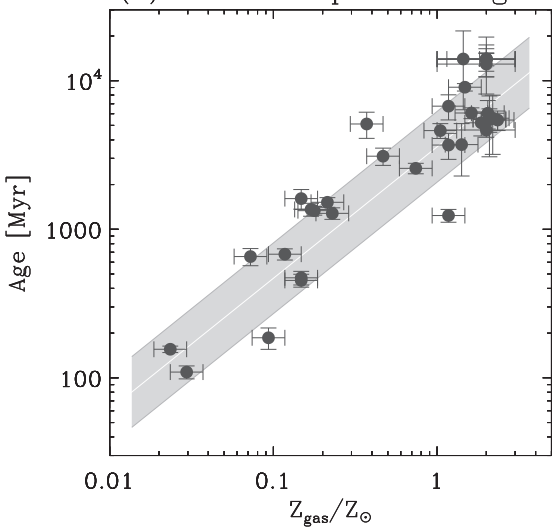

(d) PAH-to-Dust Mass Ratio

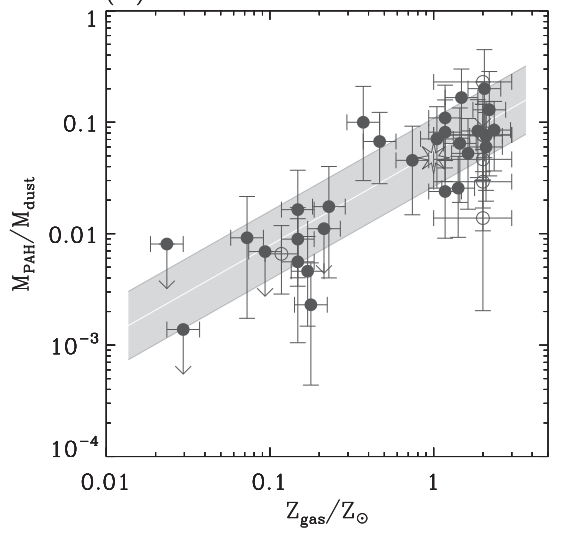

Fig. 1. (a) Ratio of the $\mathrm{IRAC}_{8} \mu \mathrm{m}$ and $\mathrm{MIPS}_{24} \mu \mathrm{m}$ broadbands of our sample, as a function of the observed metallicity. For low PAH-to-continuum ratios, the $\mathrm{IRAC}_{8} \mu \mathrm{m} / \mathrm{MIPS}_{24} \mu \mathrm{m}$ saturates. (b) Modelled contribution of $\mathrm{H}$ II regions to the IR luminosity, as a function of the observed metallicity. (c) Modelled galaxy age (from stellar population synthesis), as a function of the observed metallicity. (d) Modelled PAH-todust mass ratio as a function of the observed metallicity. Those 4 panels all come from Galliano et al. (2008). On each panel, the grey stripe shows the linear correlation to the data points $\pm 1 \sigma$.

than in normal metallicity galaxies (Madden et al. 1997). The PAHs could simply be destroyed on large scales in low-metallicity environments, due to the lower opacity of their dust depleted interstellar medium (ISM). This explanation was supported by the anticorrelation of the PAH-to-continuum

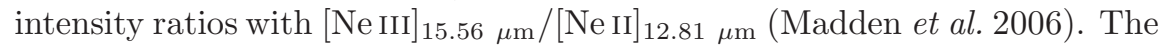
latter line ratio is a tracer of the very young, ionizing stellar populations. 
2. Alternatively, O'Halloran et al. (2006) have proposed that PAHs could be destroyed by the numerous supernova (SN) shock waves sweeping the ISM of dwarf galaxies. However, observations of Galactic supernova remnants demonstrate that the blast waves destroy all dust species (PAHs and the other grains; e.g. Reach et al. 2002) and therefore they do not pose a straightforward explanation for the selective destruction of the PAHs.

3. Finally, Dwek (2005) noted that the contribution to the enrichment of the ISM by the stellar progenitors commonly associated to PAH production, the AGB stars, was also a rising function of metallicity. Therefore the trend discussed in this paper could originate in the fact that PAHs are less abundantly produced in chemically young systems.

Correlation is not causality. Since the different mechanisms invoked above are a function of metallicity, it is difficult to find which one is at the origin of the trend. Looking at only one of these effects independently will always bias the conclusion. To really tackle the origin of this trend, it is needed to confront these various possible processes, and quantify their respective contributions.

\section{An estimate of the PAH content of galaxies}

To address this issue, we have studied the competition of these various effects within a sample of 35 nearby galaxies $\left(1 / 50 Z_{\odot} \lesssim Z_{\text {gas }} \lesssim 2 Z_{\odot}\right)$. We modelled the global UV-to-radio spectral energy distribution (SED) of each galaxy, in order to derive its PAH and dust-to-gas mass ratios. This modelling was done self-consistently, taking into account realistic dust properties, consistent stellar evolution and PAH destruction in $\mathrm{H}$ II regions. The detail of this modeling is presented by Galliano et al. (2008).

The contribution of H II regions to the IR luminosity is shown in Figure 1b. This contribution is constrained by the observations of the radio free-free and midIR thermal continua. We assume that PAHs are fully depleted in H II regions. It therefore quantifies the effect of PAH destruction on the total SED. This effect is important when considering its impact on the $\mathrm{IRAC}_{8} \mu \mathrm{m} / \mathrm{MIPS}_{24} \mu \mathrm{m}$ ratio, but is very limited on the $\mathrm{IRAC}_{8 \mu \mathrm{m}} / \mathrm{MIPS}_{160 \mu \mathrm{m}}$ which is the main constraint on the average $\mathrm{PAH}$ mass fraction.

Figure 1c shows the galaxy age, derived from stellar population synthesis and fit to the near-IR, as a function of metallicity. It demonstrates that our derived star formation history is consistent with the independent metallicity estimate of the system. This stellar population modelling is important for the subsequent chemical evolution modelling.

Finally, Figure 1d shows the evolution with metallicity of our derived PAH-todust mass ratio. Although there is a lot of scatter (partly due to the propagation of the observational uncertainties through the model), this panel shows a clear, relatively smooth, increase with metallicity of the PAH mass fraction. 


\section{A global point view on PAH evolution in galaxies}

To interpret the trend in Figure 1d, we developed a dust evolution model, that takes into account the metal and dust enrichment of the ISM by stars (Galliano et al. 2008). Assuming a continuous star formation history (following a SchmidtKennicutt law), the model predicts at each time the injection rate of the various elements and dust species. In particular, it computes the evolution of the carbon and silicate dust as a function of metallicity. We independently track the dust production associated with massive and AGB stars. We consider the destruction rate of dust in the ISM by SN blast waves to be proportional to the SN rate.

Figure 2 compares the observed trends of PAH and dust-to-gas mass ratios, with metallicity, to the theoretical evolution of carbon dust produced by AGB stars and dust produced by massive stars. The PAH evolution is strikingly coincident with the production of carbon dust by low-mass stars (see Cherchneff in this volume, for a discussion on PAH condensation in AGB stars). This comparison therefore suggests that the main origin of the $\mathrm{PAH}$ trend with metallicity is a result of the delayed injection of these molecules by AGB stars. Indeed, AGB stars start enriching the ISM after their death, $\simeq 400 \mathrm{Myr}$ after the beginning of the star formation process, when the galaxy has already been enriched by massive stars.

The stellar origin of dust is widely debated (Draine 2009, for a review). Dust is heavily processed in the ISM and reforms in dense clouds. A part of the observed ISM dust content probably does not have a stellar origin. Although our model accounts for the production of dust by various stellar progenitors, there is no assumption made on the actual location and mechanism of condensation. The comparison of our theoretical dust evolution trend to the PAH abundance in galaxies (Fig. 2) would not suffer if the actual dust condensation took place a few $10^{7}$ yr after the metal injection in the ISM. Consequently, our scenario is not in contradiction with dust reformation in the ISM, as long as it occurs on short timescales after the element injection. Dust precursors formed in the stellar envelopes could be injected in the ISM. Although their mass could account for only a few percent of the ISM budget, their presence in the ISM could be crucial to accrete more material and grow the observed ISM grains. That would explain why the dust abundance in galaxies would be closely related to the injection of those grain seeds.

Another concern on these trends is the fact that many dwarf galaxies harbour an old stellar population. It is an indication that these objects had a complex star formation history, and that they are older than indicated by their metallicity. However, the contribution of this old stellar population to the enrichment of the ISM is not significant, otherwise their metallicity would be higher. Moreover, this stellar population does not dominate the integrated SED of these objects (Fig. 1b). Finally, Lee et al. (2009) studied a sample of local dwarf galaxies and showed that the star formation bursts were only responsible for about a quarter of the total star formation in the overall population. 


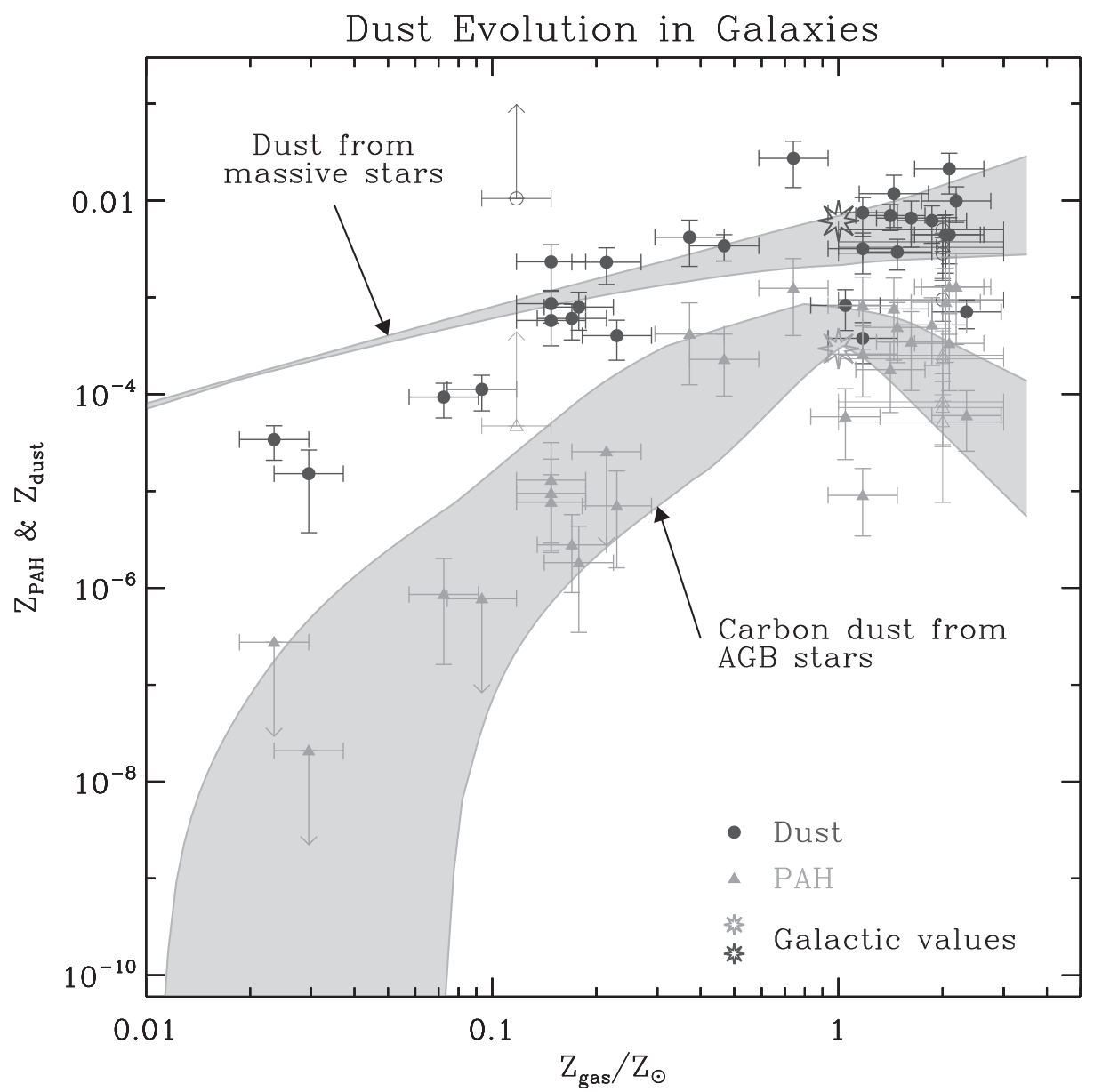

Fig. 2. Comparison between our dust evolution model and the derived trend of PAH and dust-to-gas mass ratios $\left(Z_{\mathrm{PAH}}=M_{\mathrm{PAH}} / M_{\text {gas }}\right.$ and $\left.Z_{\text {dust }}=M_{\text {dust }} / M_{\text {gas }}\right)$ with the observed gas metallicity. The grey envelopes show the result of our dust evolution model. The various curves within the envelopes represent the spread caused by assuming different star formation rates and different dust destruction efficiencies by SN blast waves. No parameter has been adjusted to fit the dust evolution trends to the observed dust content.

\section{Additional confirmations from spatially resolved studies}

Although our global approach is relatively crude, because of its lack of spatial resolution, it provides general trends of the various processes and allows a comparison of their respective contributions. Several studies have addressed some of these issues by looking at spatially resolved observations. 
Muñoz-Mateos et al. (2009) by looking at radial trends within spiral galaxies confirmed the relation between PAH and the evolution of AGB stars. They even found indications of the reversing of the trend at high metallicity, explained by the fact that the enrichment becomes dominated by O-rich low-mass stars (Fig. 2).

In the Large Magellanic Cloud (LMC), the spatial distribution of the PAH mass fraction is associated with the stellar bar (Paradis et al. 2009). It has been independently confirmed by Meixner et al. (2010). However, this is not the case in the Small Magellanic Cloud $\left(\mathrm{SMC} ; \simeq 1 / 6 Z_{\odot}\right.$; Sandstrom et al. 2010, also in this volume). In the SMC, the PAHs appear to be massively depleted in the diffuse ISM, but they are abundant in PDRs, although their abundance is significantly lower than in the Milky Way $(\simeq 0.2-0.4$ Galactic PAH abundance). Consequently, this study shows, with spatial resolution, that despite the photodestruction effects dominate in the diffuse ISM, the PAHs are underabundant even in regions where they are shielded from the hard radiation field. It therefore supports the idea that the photodestruction processes are not sufficient to account for the trend of PAH abundance with metallicity, and that the deficiency of their production has to be invoked.

\section{References}

Draine, B.T., 2009, ed. in T. Henning, E. Grün, \& J. Steinacker, Astron. Soc. Pac. Conf. Ser., 414, 453

Dwek, E., 2005, ed. in C.C. Popescu, \& R.J. Tuffs, AIP Conf. Proc. 761: The Spectral Energy Distributions of Gas-Rich Galaxies, 103

Engelbracht, C.W., Gordon, K.D., Rieke, G.H., et al., 2005, ApJ, 628, L29

Galliano, F., Dwek, E., \& Chanial, P., 2008, ApJ, 672, 214

Lee, J.C., Kennicutt, R.C., José G. Funes, S.J., et al., 2009, ApJ, 692, 1305

Madden, S.C., Galliano, F., Jones, A.P., \& Sauvage, M., 2006, A\&A, 446, 877

Madden, S.C., Poglitsch, A., Geis, N., et al., 1997, ApJ, 483, 200

Meixner, M., Galliano, F., Hony, S., et al., 2010, A\&A, 518, L71

Muñoz-Mateos, J.C., Gil de Paz, A., Boissier, S., et al., 2009, ApJ, 701, 1965

O'Halloran, B., Satyapal, S., \& Dudik, R.P., 2006, ApJ, 641, 795

Paradis, D., Reach, W.T., Bernard, J., et al., 2009, AJ, 138, 196

Reach, W.T., Rho, J., Jarrett, T.H., \& Lagage, P.-O., 2002, ApJ, 564, 302

Sandstrom, K.M., Bolatto, A.D., Draine, B.T., et al., 2010, ApJ, 715, 701 